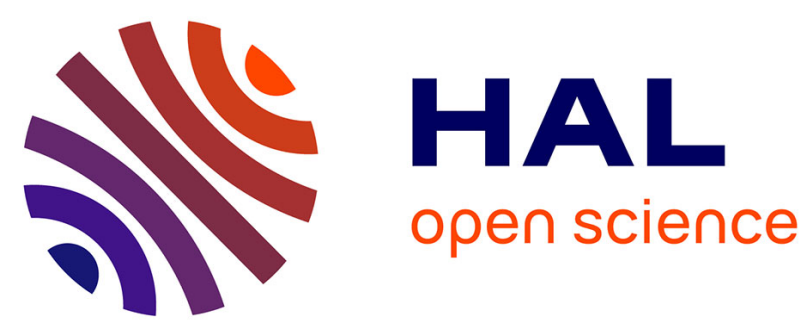

\title{
iTowns, le nouveau moteur de visualisation 3D de données géospatiales du Géoportail
}

\author{
Mirela Konini, Alexandre Devaux, Mathieu Brédif
}

\section{To cite this version:}

Mirela Konini, Alexandre Devaux, Mathieu Brédif. iTowns, le nouveau moteur de visualisation 3D de données géospatiales du Géoportail. Annales des mines - Série Responsabilité et environnement, 2019, Cartographie 4.0: naviguer avec les cartes du XXIe siècle, Nº 94, pp.14-18. 10.3917/re1.094.0014 . hal-02543879

\section{HAL Id: hal-02543879 \\ https://hal.science/hal-02543879}

Submitted on 15 May 2020

HAL is a multi-disciplinary open access archive for the deposit and dissemination of scientific research documents, whether they are published or not. The documents may come from teaching and research institutions in France or abroad, or from public or private research centers.
L'archive ouverte pluridisciplinaire HAL, est destinée au dépôt et à la diffusion de documents scientifiques de niveau recherche, publiés ou non, émanant des établissements d'enseignement et de recherche français ou étrangers, des laboratoires publics ou privés. 


\author{
Par Mirela KONINI \\ IGN \\ Alexandre DEVAUX \\ et Mathieu BRÉDIF \\ Université Paris-Est, LASTIG GEOVIS, IGN, ENSG
}

\begin{abstract}
La visualisation est un mode privilégié de l'interaction des utilisateurs avec l'information géographique, et sa représentation efficace est d'autant plus importante que les données sont massives et hétérogènes et que les utilisateurs et les usages sont variés. Au-delà de la visualisation de données 2D sur un écran ou une carte papier, la visualisation de données 3D pose de nouveaux défis et nécessite des outils appropriés : volume des données, multiplicité des formats, stylisation et gestion des parties visibles, modes d'interaction et de navigation...

iTowns est une plateforme technologique de l'IGN qui permet de visualiser des données géographiques 3D via le Web et propose des fonctions d'interaction avancées dans un environnement métrologique.

Initialement développé par les laboratoires de recherche de l'IGN comme un outil de visualisation de données images et LiDAR issues de la cartographie mobile (c'est-à-dire acquises au moyen d'un véhicule équipé de capteurs), iTowns a évolué et permet aujourd'hui de naviguer de façon immersive au sein d'un très grand volume de données 3D, et ce dans toute la gamme d'échelles, depuis l'espace jusqu'au sol. Des interfaces sont également disponibles pour la manipulation de ces données. Désormais moteur de visualisation 3D du Géoportail (1), iTowns s'enrichit continuellement de nouvelles fonctionnalités : en sus de la visualisation en 3D du territoire pour le grand public, il permet de développer des applications Web à usage professionnel pour co-visualiser différents types de données, les annoter, procéder à des analyses, des mesures...
\end{abstract}

\section{Une plateforme innovante issue de la recherche de I'IGN}

À l'origine, iTowns était un projet de recherche qui, lancé en 2008 et financé par l'ANR, visait à proposer un outil de navigation immersive via le Web au sein de données images et LiDAR issues de capteurs montés sur une plateforme mobile géoréférencée - le véhicule de cartographie mobile Stéréopolis de l'IGN permet en effet d'acquérir différents types de données géolocalisées au moyen des divers capteurs qu'il embarque (photographies numériques, laser, capteurs thermiques, etc.). Développé de 2008 à 2011 par le laboratoire de recherche Matis de I'IGN, le projet iTowns proposait une visualisation dans le navigateur des résultats des algorithmes automatiques d'extraction d'informations contenues dans des images (détection de marquages au sol, panneaux, textes, végétations).
Par la suite, le produit iTowns a été impliqué dans de multiples projets de recherche, notamment le projet FUI Terra Mobilita, dont le but était d'améliorer la génération de cartes de précision centimétrique au niveau de la rue dans le cadre d'études d'accessibilité pour les personnes à mobilité réduite (PMR). L'idée était d'utiliser iTowns pour visualiser la rue reconstituée à partir de données acquises de façon dématérialisée par un véhicule de cartographie mobile afin de réaliser, à moindre coût, des études de diagnostic d'accessibilité pour les PMR : possibilité grâce à cet outil de naviguer virtuellement, de réaliser des mesures et d'annoter les images directement à partir d'un navigateur Web (voir la Figure 1 de la page suivante). Cela

(1) L'infrastructure d'hébergement et de diffusion en ligne de données géographiques mise en œuvre par l'IGN pour le compte de l'État: http://www.geoportail.gouv.fr 


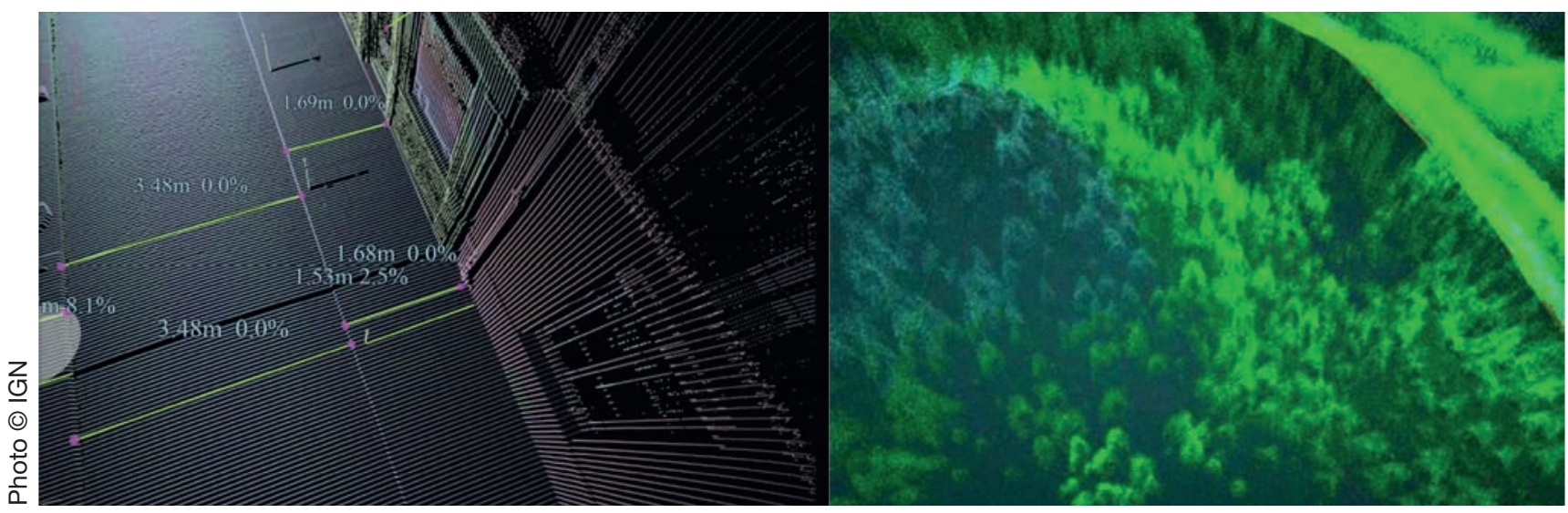

Figure 1 : Co-visualisation de données images et LiDAR et réalisation de mesures précises lors de l'étude de l'accessibilité de l'espace urbain pour les personnes à mobilité réduite (à gauche, le projet FUI Terra Mobilita). Visualisation du LiDAR aérien de masse dans le cadre de l'étude de l'évolution de la canopée forestière (à droite, le projet ANR Foresee).

permettait d'éviter une collecte fastidieuse d'informations directement sur le terrain.

En parallèle, I'IGN a participé au projet ANR Foresee : il a notamment développé un démonstrateur basé sur iTowns. II s'agissait de co-visualiser des nuages de points LiDAR résultant d'une acquisition aérienne à l'échelle d'un département et des données orthophotographiques ${ }^{(2)}$ et MNT ${ }^{(3)}$ pour étudier l'évolution de la canopée et des espèces forestières. Le défi était d'explorer des milliards de points LiDAR via un navigateur Web sur un fond de carte 3D issu d'un terrain modélisé par un MNT et texturé par une orthophotographie (voir la Figure 1 ci-dessus). Cela a permis à I'IGN de créer par la suite de nouvelles bases de données LiDAR pour procéder à un affinage (jusqu'à 1 mètre de précision) du MNT français et de mettre en place de nouvelles collaborations pour réaliser d'autres applications, notamment en foresterie.

iTowns a su mettre à profit l'arrivée des technologies WebGL et être supporté par les navigateurs Internet (à partir des années 2010) permettant l'utilisation directe de la carte graphique à partir d'une application Web. Cette technologie WebGL permet à la plateforme iTowns d'afficher de grandes quantités d'informations 3D, comme les nuages de points LiDAR et les surfaces maillées en 3D, dans un navigateur Internet, avec des performances similaires à celles d'un jeu vidéo.

Une des forces de l'application est la liberté de navigation qu'elle offre, le point de vue n'est pas restreint aux positions des prises de vue initiales : en effet, grâce au rendu projectif, il est possible de naviguer librement et de manière continue en trois dimensions, de l'espace à la rue, et ce, en choisissant le point de vue que l'on souhaite tout en conservant la précision originale des acquisitions.

Les projets d'innovation dans lesquels iTowns a été impliqué ainsi que l'arrivée des nouvelles technologies ont façonné la plateforme et enrichi ses capacités. C'est l'ensemble de ces capacités - la co-visualisation de données massives et hétérogènes, l'interaction avec les données et la possibilité de faire diverses simulations - qui constitue aujourd'hui l'ADN d'iTowns et le distingue fortement d'une application de type street view.

\section{Une industrialisation réussie}

Tirant parti de huit années de recherche ainsi que du développement de nombreux démonstrateurs basés sur la technologie iTowns, I'IGN a décidé, en 2016, d'industrialiser la plateforme par un transfert partiel des équipes de Recherche pour renforcer celles en charge des développements avec, comme premier objectif, de devenir le moteur de visualisation 3D du Géoportail. L'industrialisation a introduit de nouvelles fonctionnalités, notamment une vision depuis l'espace en 3D et la possibilité de co-visualiser tout type de données géospatiales, des images satellitaires couvrant le globe terrestre aux nuages de points de précision centimétrique au niveau de la rue, que ces données soient produites par I'IGN ou par des acteurs tiers. L'un des principaux verrous technologiques à lever était d'arriver à gérer un très grand volume de données tout en offrant une très grande amplitude de variation de l'échelle de visualisation, le tout en garantissant la stabilité du produit pour un usage ouvert au grand public.

Ce défi a été relevé en 2017, faisant d'iTowns le moteur permettant de naviguer en 3D à l'échelle de tout le territoire : il est capable de co-visualiser en 3D, sans qu'il y ait besoin de plugin, les données orthophotographiques, les données vecteurs, les modèles numériques de terrain et de surface (MNT et MNS ${ }^{(4)}$ ) de I'IGN, des modèles 3D à différents niveaux de détail (LoDs, pour Level of Details), servis par des flux standards (WMS ${ }^{(5)}$, WMTS ${ }^{(6)}$, WFS ${ }^{(7)}$ et 3 DTiles $\left.{ }^{(8)}\right)$, avec des mises à jour en continu.

(2) Orthophotographie : image couleur superposable à une carte, recomposée à partir d'images aériennes rectifiées géométriquement. (3) Modèle numérique du terrain : carte d'altitude du sol, sans sursol (arbres, bâtiments...).

(4) Modèle numérique de surface.

(5) Web Map Service, service HTTP qui fournit des images géoréférencées.

(6) Web Map Tile Service, service HTTP qui fournit des images géoréférencées tuilées.

(7) Web Feature Service, protocole d'échange de données vecteurs qui permet de connaitre les structures et les sources de la donnée spatiale.

(8) Structure d'organisation des données géospatiales 3D en arborescence tuilée pour être diffusées en flux. 


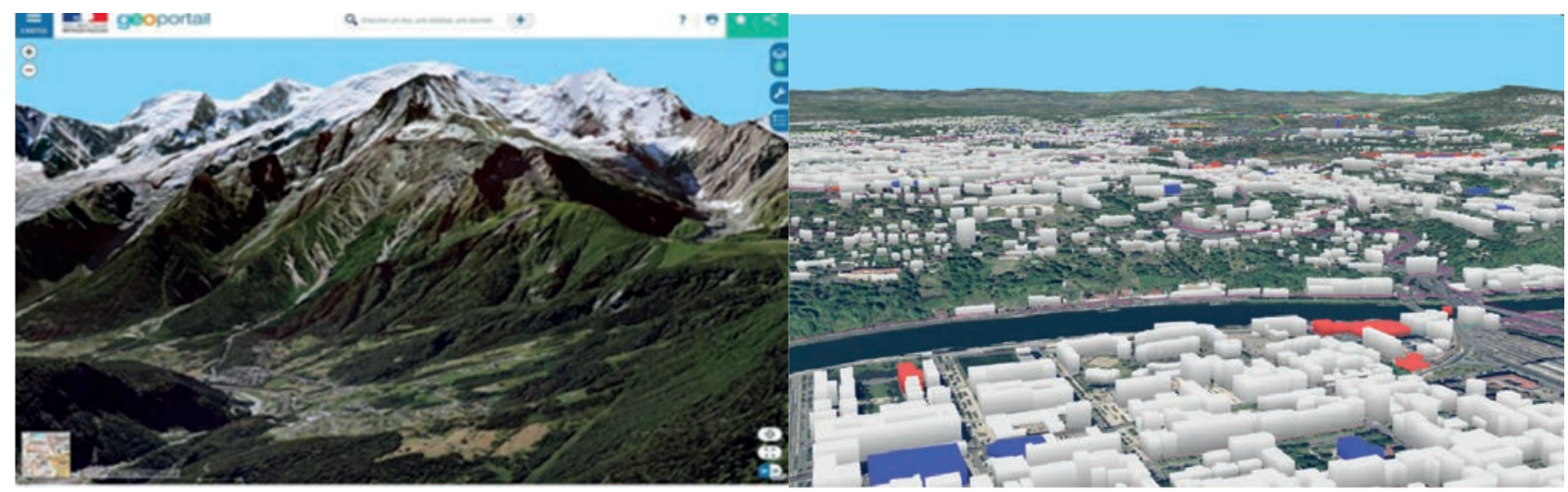

Figure 3 : Le moteur de visualisation 3D du Géoportail : https://www.geoportail.gouv.fr/

Au-delà de l'utilisation d'iTowns comme solution de visualisation 3D pour le Géoportail, de multiples applications et services ont été développés pour répondre aux besoins nés de la conduite des projets de l'IGN ou de ceux de ses partenaires et clients.

\section{Applications industrielles réalisées avec iTowns}

\section{Visite virtuelle en milieux complexes}

Au cours de leurs interventions, les égoutiers sont exposés à des risques liés à l'insalubrité. Ces visites doivent donc être réduites en durée, d'où l'intérêt de les préparer en amont. C'est dans cet objectif que I'IGN a participé à une expérimentation visant à la numérisation du réseau des égouts de la ville de Paris pour réaliser ultérieurement des visites virtuelles. Des données images ont été acquises grâce à un système composé de quatre caméras légères ${ }^{(9)}$ prototypées au sein de l'IGN et montées sur un sac à dos. La reconstruction d'un modèle 3D a été faite automatiquement à partir des images orientées (en position et en rotation) produites dans la phase d'acquisition en utilisant la solution photogrammétrique MicMac ${ }^{(10)}$. Un nuage de points dense est généré à partir de ces images, puis un maillage. Les images orientées sont ensuite projetées à la volée sur le maillage généré. II est ainsi possible de naviguer en immersif dans le modèle, de prendre des mesures et/ou de procéder à diverses simulations (aménagement de matériel, étude de la mobilité d'un avatar humain, etc.) depuis la rue ou directement dans les souterrains, en continu.
La simulation de phénomènes (la montée des eaux, par exemple)

La simulation est faite sur la base du modèle numérique altimétrique précis continu terre-mer, Litto3D ${ }^{(11)}$, dont la précision altimétrique pour le littoral français est de 20 centimètres. Cela permet de faire une première analyse des zones à risque et de prendre les mesures adéquates en matière d'aménagement, de sécurisation des points sensibles et de protection des habitations.

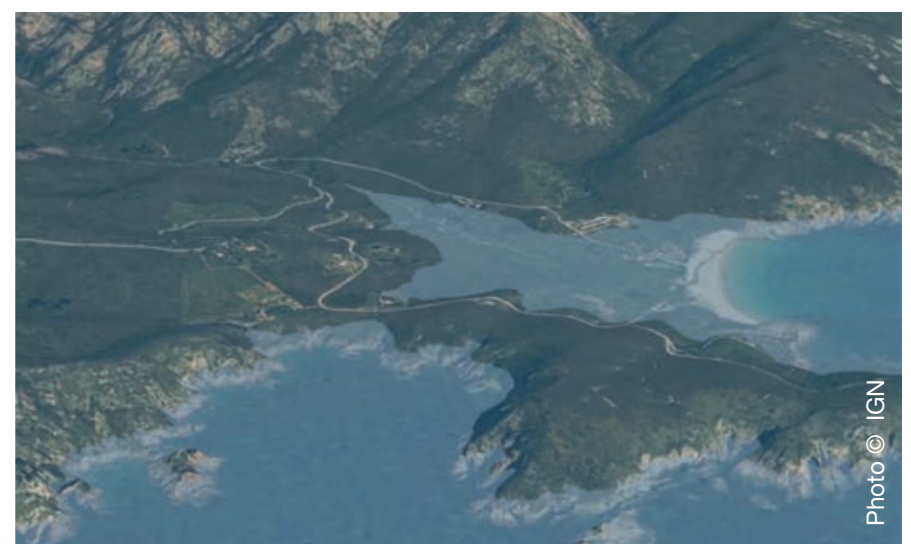

Figure 5 : Simulation de la montée des eaux sur le littoral corse.

(9) La caméra légère est conçue par le laboratoire d'opto-électronique, de métrologie et d'instrumentation (LOEMI) de l'IGN. (10) MicMac est un logiciel libre de photogrammétrie qui a été développé par I'IGN. II permet de reconstruire des modélisations 3D à partir d'un ensemble d'images : http://logiciels.ign.fr/?Micmac

(11) Litto3D ${ }^{\circledR}$ est un modèle numérique altimétrique précis continu terre-mer réalisé en commun par le SHOM et l'IGN : http://Www. professionnels.ign.fr/litto3d
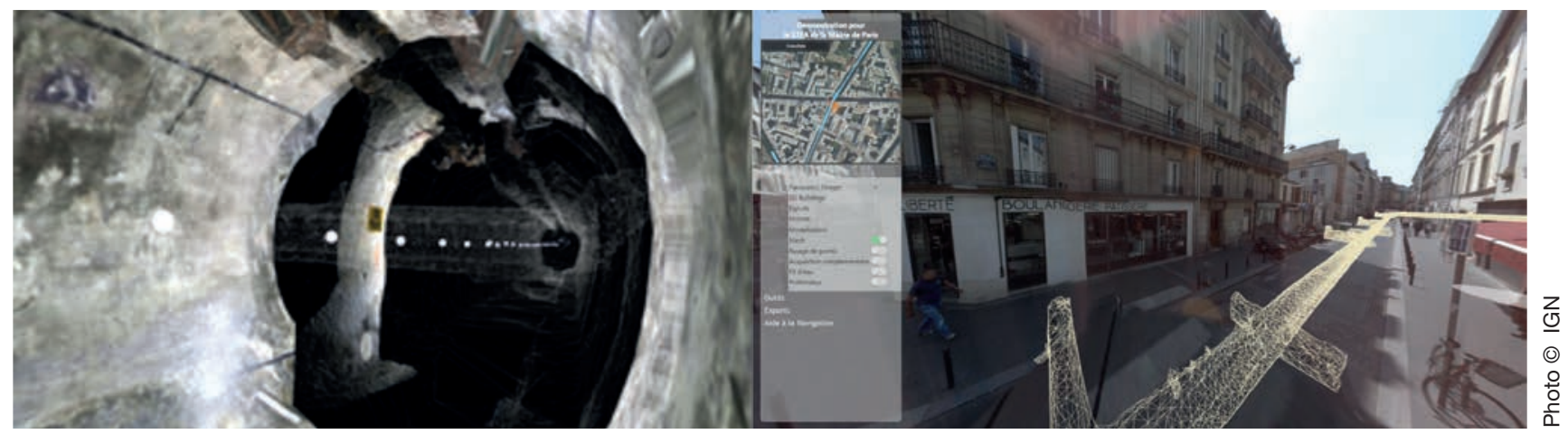

Figure 4 : Visite virtuelle dans les égouts de Paris. 

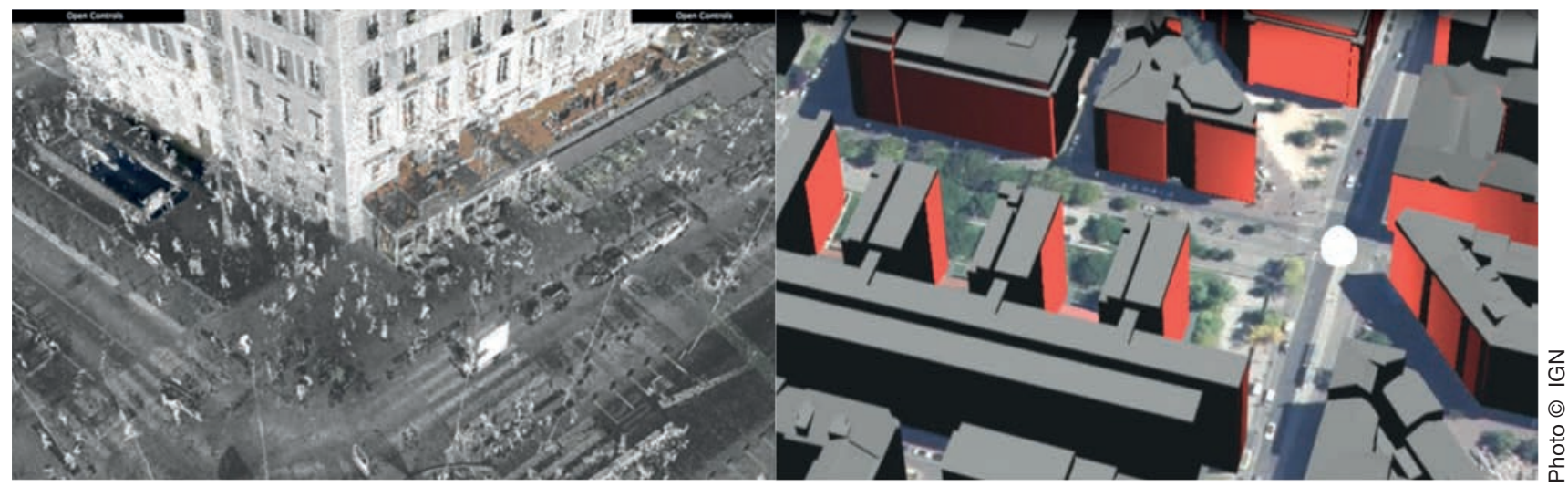

Figure 6 : Visualisation d'images LiDAR terrestres de la ville de Marseille (à gauche). Sécurisation d'un parcours basée sur le calcul des inter-visibilités. Les plans apparaissant en rouge représentent les zones visibles depuis la cible symbolisée par la sphère blanche lorsqu'elle se déplace, ou, inversement, les zones à partir desquelles il est possible de voir la cible.

\section{Sécuriser un site ou un parcours}

La sécurisation des établissements recevant du public est un des enjeux majeurs pour pouvoir préserver l'intégrité physique de leurs occupants. Grâce à la co-visualisation des données images et LiDAR de l'intérieur comme de l'extérieur d'un bâtiment, il est possible de connaître avec précision ses différents points d'accès afin de le sécuriser, de préparer un plan d'intervention ou de gérer une crise à distance.

\section{Visualisation des modèles 3D des villes}

Les villes intelligentes, dites smart cities, sont dotées de différents types de capteurs de données fournissant des informations permettant de gérer efficacement leurs actifs et leurs ressources. Le modèle $3 \mathrm{D}$ de la ville est le point pivot de la ville intelligente, car il contient des informations sur l'infrastructure de base qu'il est possible de compléter en ajoutant d'autres données comme les systèmes de transport, les réseaux de distribution d'eau et d'énergie, le bruit, la pollution, etc. iTowns aide à comprendre et à interpréter ces données ; il permet aussi de faire des simulations de l'aménagement de bâtiments ou de modèles urbains. II est donc possible de construire des applications qui aident les décideurs lors de la mise en œuvre des plans d'aménagement urbain ou qui facilitent la concertation citoyenne lorsque ces plans sont soumis à consultation.

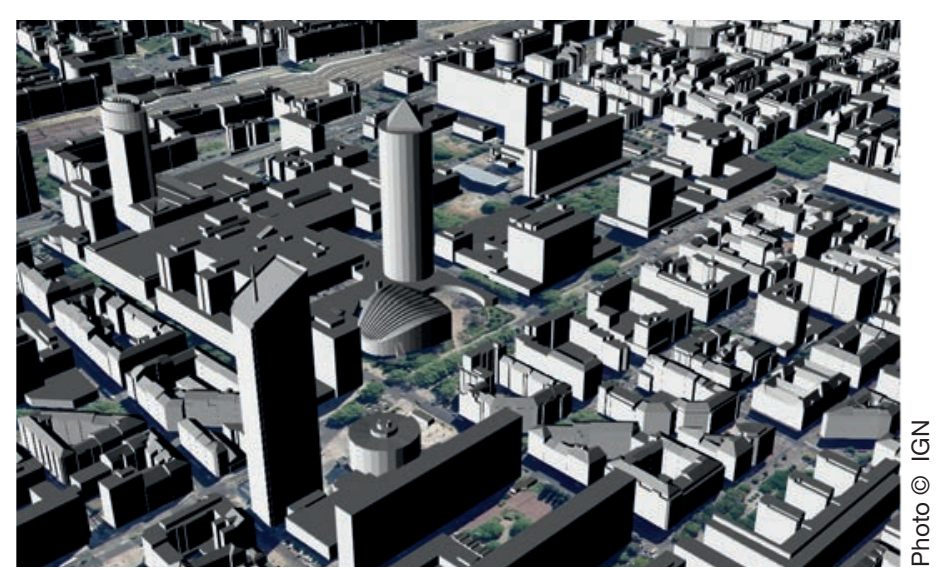

Figure 7 : Visualisation du modèle 3D de la ville de Lyon à partir de données ouvertes (open data) du Grand Lyon. Le survol avec la souris permet d'afficher les métadonnées des bâtiments.

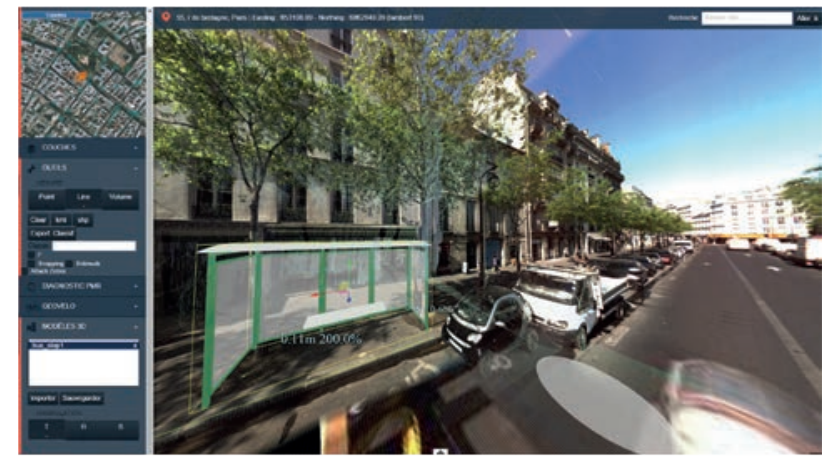

Figure 8 : Simulation d'aménagement du mobilier urbain (le positionnement et l'échelle du modèle sont modifiables lors de la simulation).

\section{Un projet ouvert et collaboratif}

iTowns est diffusé sous licence libre (Licences CeCILL-B \& MIT), c'est le seul produit français de cette envergure à être diffusé en open source ${ }^{(12)}$. Cette décision prise en 2016 par I'IGN correspond à sa stratégie de faciliter l'utilisation de ses référentiels géographiques, de partager ses outils et son expertise et de créer une communauté de développeurs qui agissent en synergie pour concevoir de nouvelles applications et enrichir la plateforme. Cela permet d'enclencher une nouvelle dynamique pour le projet, car il est depuis lors ouvert aux contributions externes : tout contributeur qui le désire peut proposer des évolutions et/ou utiliser la plateforme pour réaliser ses propres applications, sans obligation d'apporter une quelconque contribution financière. La mise en open source de la plateforme iTowns permet à l'IGN de faciliter la coopération autour de cette plateforme dans le cadre de projets de recherche et d'innovation avec ses partenaires académiques ou industriels, mais aussi à des fins d'éducation.

\section{Un socle pour les travaux de recherche}

iTowns ayant atteint un niveau de maturité technologique élevé, il est dorénavant possible de l'utiliser directement dans des projets de recherche et de créer des prototypes

(12) http://www.itowns-project.org/ 
rapidement. Basé sur la librairie open source Web3D ThreeJS. la plus développée au monde, iTowns permet le prototypage extrêmement rapide de projets R\&D. Différents projets de recherche français et européens (Alegoria, URCLIM) s'appuient sur iTowns pour créer de nouveaux modes de navigation et d'interaction. Dans le projet ANR Alegoria (http://alegoria.ign.fr), iTowns permettra la navigation en $3 \mathrm{D}$ au sein de grands corpus de données iconographiques anciennes à partir de points de vue aériens et terrestres, de façon continue. Le projet ERA4CS URCLIM (http://urclim.prod.lamp.cnrs.fr), visant à la création de nouveaux services climatiques, utilisera iTowns pour permettre la dissémination de résultats de modèles climatiques. L'application doit jouer un rôle primordial afin d'adapter le rendu à chaque utilisateur, que ce soit un décideur public, un citoyen ou un scientifique. C'est aussi un excellent outil d'enseignement dans le monde géospatial.

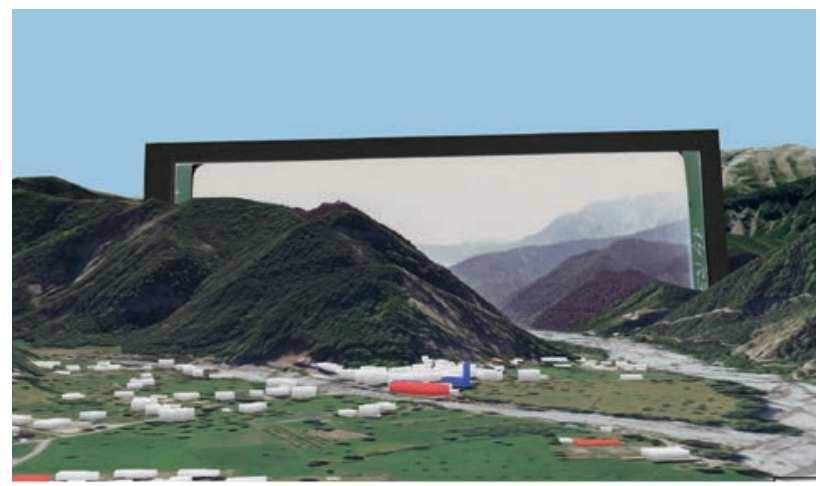

Figure 9 : Photographie ancienne retravaillée dans iTowns (projet ANR Alegoria). Archives nationales/Fonds LAPIE - (C) IGN 2019.

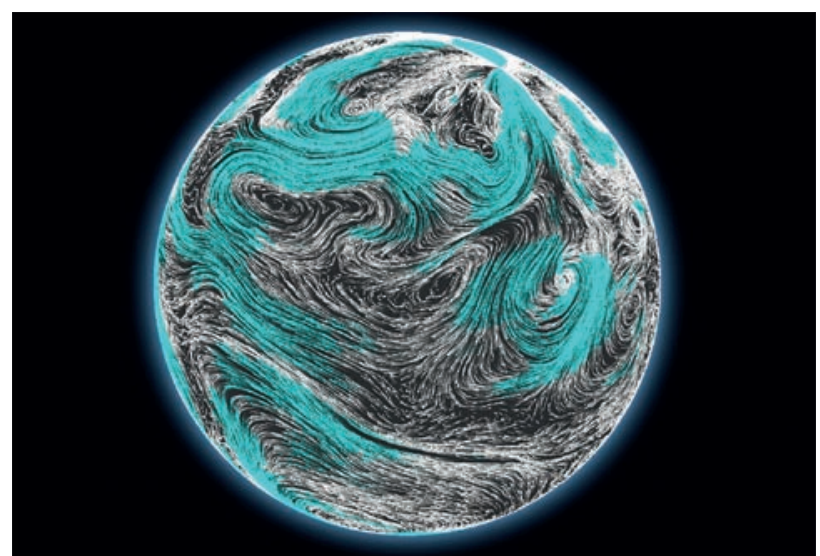

Figure 10 : Visualisation de flux d'air (travaux préliminaires au projet ERA4CS URCLIM).

\section{Une brique pour la future Géoplate- forme}

Les capacités de la plateforme iTowns et l'interopérabilité des données permettent de co-visualiser les référentiels de I'IGN et d'autres sources de données provenant de ses partenaires. Selon le contexte et le souhait de ces derniers, I'IGN pourra prendre en charge l'hébergement et la diffusion de leurs données. Les données au format standard (CityGML) sont intégrées dans la base, stockées dans les entrepôts de I'IGN et converties dans un format adéquat pour être diffusées en flux (3DTiles). Le partenaire

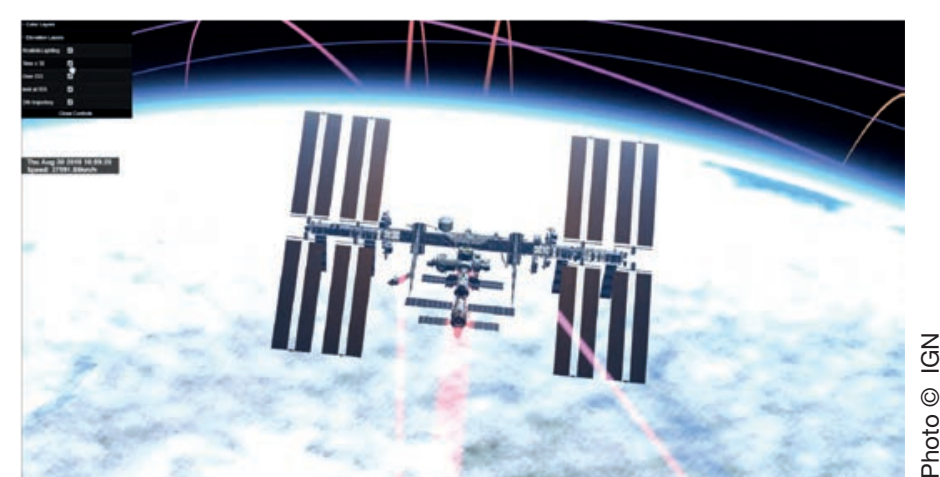

Figure 11 : Visualisation d'orbites de satellites.

pourra mettre à jour cette base de données directement de chez lui ou via l'infrastructure de l'IGN, favorisant ainsi la mutualisation de la collecte et de la mise à jour des données. C'est le principe retenu pour la Géoplateforme de I'IGN qui est actuellement en cours de construction, et dont iTowns constitue l'une des briques.

\section{Bibliographie}

CARAFFA L., BRÉDIF M. \& VALLET B. (2011), "3D octree based watertight mesh generation from ubiquitous data", International Archives of Photogrammetry Remote Sensing and Spatial Information Sciences (GeoBigData), volume $\mathrm{XL}-3 / \mathrm{W} 5$.

DEVAUX A., PAPARODITIS N. \& BRÉDIF M. (2012), “A web-based $3 d$ mapping application using webgl allowing interaction with images, point clouds and models", International Conference on Advances in Geographic Information Systems (20 th $A C M$ SIGSPATIAL GIS 2012), Redondo Beach, CA (USA).

VANDERGUCHT D., PAPARODITIS N., DEVAUX A., BÉNARD M. \& MALLET C. (2015), « ZForest : un prototype de plateforme Web de visualisation LiDAR, raster et vecteur de grande échelle ", Revue française de photogram-

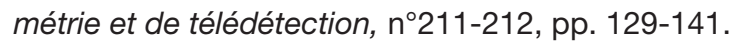

MASSE A. \& CHRISTOPHE S. (2015), «Visualisation homogène du littoral à partir de données géographiques hétérogènes spatio-temporelles ", Actes du Colloque International de Géomatique et d'Analyse Spatiale (SAGEO 2015).

DEVAUX A. \& BRÉDIF M. (2016), "Realtime projective multi-texturing of point clouds and meshes for a realistic street-view web navigation", Web3D '16 Proceedings of the $21^{\text {st }}$ International Conference on Web3D Technology, pp. 105-108.

PICAVET V., BRÉDIF M., KONINI M. \& DEVAUX A. (2016), « iTowns, framework web pour la donnée géographique 3D », $X Y Z, n^{\circ} 147,2^{\text {ème }}$ trimestre, pp. 49-52.

CARAFFA L., BRÉDIF M. \& VALLET B. (2017), “3D watertight mesh generation with uncertainties from ubiquitous data", Asian Conference on Computer Vision (ACCV), novembre 2016, Taipei (Taiwan), pp. 377-391.

RUPNIK E., DAAKIR M. \& PIERROT-DESELIGNY M. (2017), "MicMac - A free, open-source solution for photogrammetry", Open Geospatial Data, Software and Standards, pp. 2-14. 